\title{
Symptomatic Solitary Osteochondroma of the Subaxial Cervical Spine in a 52-Year-Old Patient
}

\author{
Vijay Sekharappa, Rohit Amritanand, Venkatesh Krishnan, Kenny Samuel David \\ Spinal Disorders Surgery Unit, Department of Orthopaedics, Christian Medical College, Vellore, India
}

Osteochondromas are the most common benign tumors of the bone. They mostly arise from the appendicular skeleton and present clinically in the second or third decade of life. Ostechondromas arising from the subaxial cervical spine and presenting after the 5th decade of life are extremely rare. We report a 52-year-old male patient who presented with numbness and subjective weakness of left upper and lower limbs and neck pain, and had lobulated bony hard fixed swelling in the right lower cervical paraspinal region. Radiological images revealed a bony swelling arising from C4 and C5 lamina with a cartilaginous cap and intraspinal extension. Excision biopsy with stabilisation of the spine was performed. Histopathalogical examination of the specimen confirmed the diagnosis of osteochondroma. We conclude surgical excision of such rare tumors, including the cartilaginous cap as well as the intraspinal component can reliably produce a good clinical outcome.

Keywords: Osteochondroma; Cervical spine; Elderly

\section{Introduction}

Osteochondromas are the most common benign tumors of the bone accounting for $10 \%$ to $15 \%$ of all bone tumors and $20 \%$ to $50 \%$ of all benign bone tumors [1]. They are most commonly located in the appendicular skeleton, in the metaphyseal region of long bones $[2,3]$. Spinal osteochondromas account for $1.3 \%$ to $4.1 \%$ of all osteochondromas $[2,4]$ and rarely cause spinal cord compression $[1,4]$. Cervical spine osteochondromas usually involve $\mathrm{C} 1$ and C2 vertebrae $[3,5]$. Ostechondromas do not grow after skeletal maturity and usually manifest clinically in the second and third decade of life $[3,5]$. Osteochondromas arising from the subaxial cervical spine (C3 and below) and producing clinical symptoms late in adult life is extremely rare. We present one such case of osteochondroma arising from the subaxial cervical spine and pro- ducing clinical symptoms in a 52-year-old male.

\section{Case Report}

A 52-year-old lawyer presented with a history of decreased sensation and subjective weakness of his left upper and lower limbs and neck pain for the previous 5 months. A swelling over the back of neck was detected by a local practitioner and referred to us for further management. Examination of the cervical spine revealed a bony hard fixed swelling over the right lower cervical paraspinal region which was non tender and had a smooth lobulated surface. Terminal ranges of neck movements were painfully restricted. Neurological examination revealed decreased sensation from C6 and below with absent superficial abdominal and cremasteric reflexes on the left side. There was no objective motor weakness in any of

Received Sep 13, 2012; Revised Oct 22, 2012; Accepted Oct 22, 2012

Corresponding author: Vijay Sekharappa

Spinal Disorder Surgery Unit, Department of Orthopaedics, Christian Medical College,

Vellore, Tamil Nadu 632004, India

Tel: +91-9025267551, Fax: +91-04162232035,E-mail: vijays_sdumc@yahoo.co.in 
the 4 limbs and no clinical signs of a cervical compressive myelopathy.

Plain radiographs, magnetic resonance imaging (MRI) and computed tomography (CT) scans of the cervical spine are shown in Figs. 1-3. In view of spinal canal encroachment, progressive sensory symptoms, and suspicion of malignant transformation due to late presentation and calcifications within the predominantly cartilaginous lesion, an excision biopsy was advised. The patient un- derwent a posterior cervical approach with exposure of the large lobulated mass extending from C2 to C6 on the left side. The lesion had a bony hard consistency in its cranial part and was firm in the lower cartilaginous portion. Lateral mass screws were inserted unilaterally on the right side from $\mathrm{C} 3-\mathrm{C} 5$. Due to its large size and its overlying and obscuring the underlying laminae, the cartilaginous lesion was first resected using osteotomes and the remainder of the lesion was then thinned down

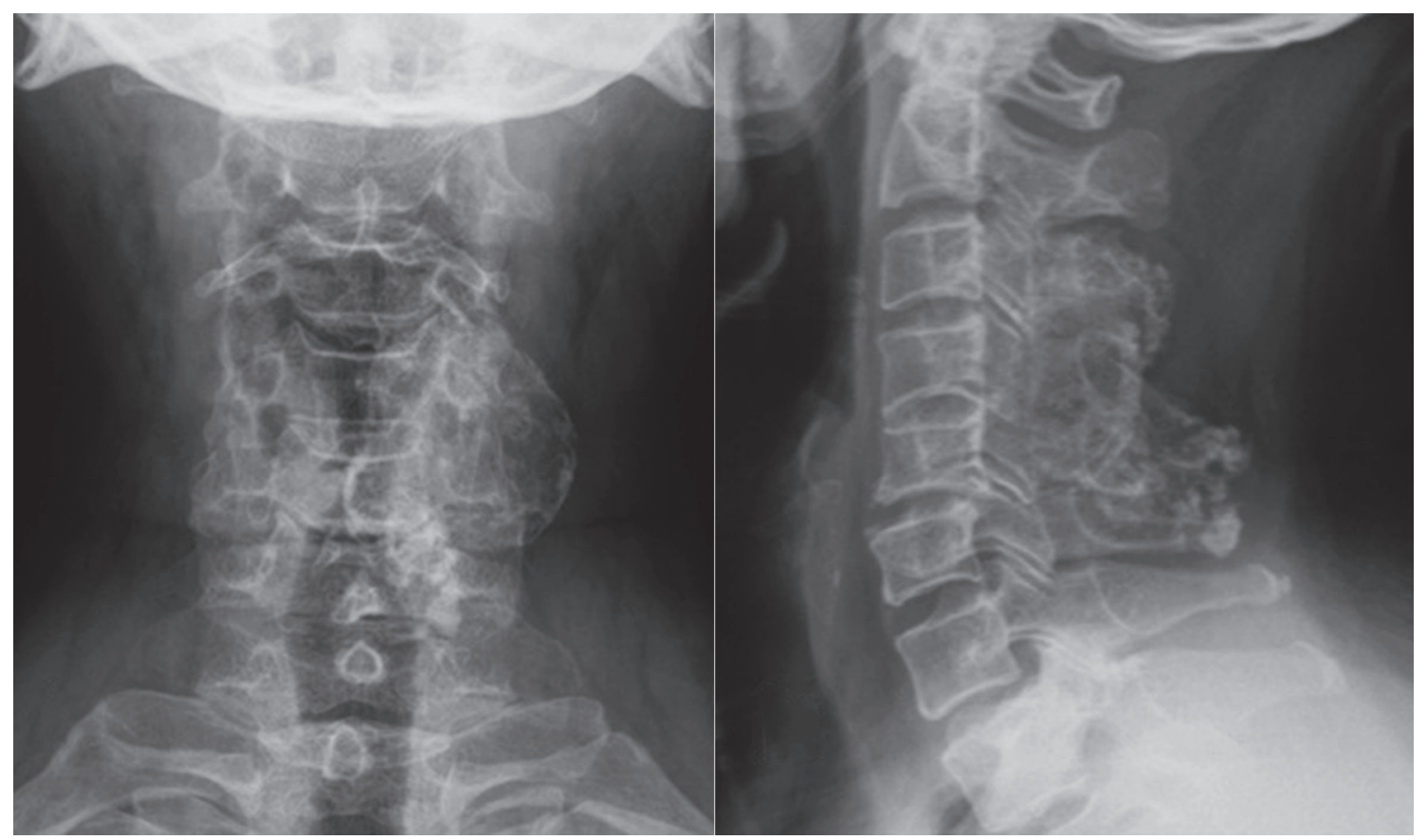

Fig. 1. X-ray of cervical spine anterior-posterior and lateral view showing a bony mass with well defined margins, extending from C2 to C6 vertebra, measuring $4 \times 3 \mathrm{~cm}$ and showing continuity with the lamina and facets of C3, C4, and C5 on the left side.
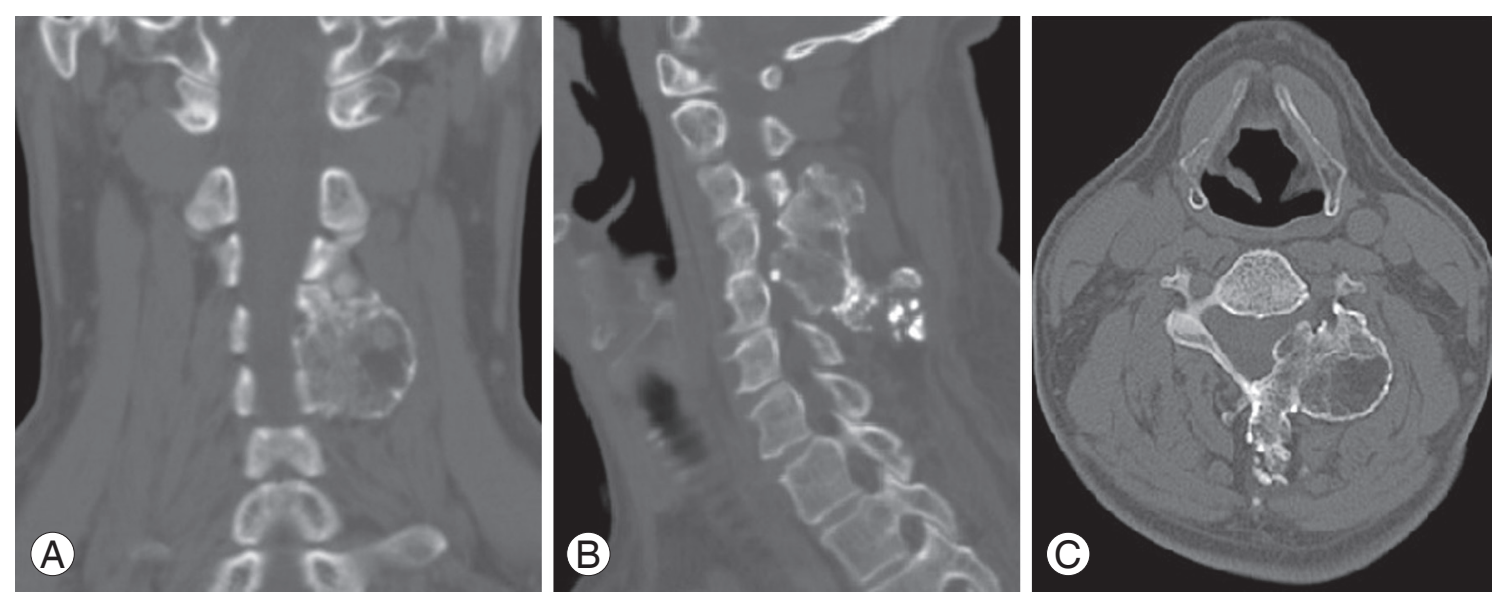

Fig. 2. Computed tomography scan. Coronal (A), saggital (B), and axial (C) images of the cervical spine showing a well-defined bony mass arising from the lamina of $\mathrm{C} 4$ and $\mathrm{C} 5$ with intraspinal extension and soft tissue calcifications in inferoposterior aspect of the tumor, representing calcification within the catilagenous cap. 
to the level of the laminae using rongeurs. The stalk was found to be attached to the C4 and C5 laminae. A C4 and C5 laminectomy was then performed and the intraspinal extension of the tumor was resected with the laminae (Fig. 4). The osseous and the cartilaginous parts were sent for histopathological examination separately and the biopsy was subsequently reported as osteochondroma with no evidence of any malignant transformation (Fig. 5).

Postoperatively the patient reported progressive improvement in numbness of left upper and lower limbs (Fig. 6). At 6 months, follow-up sensations over left limbs had recovered completely and a repeat X-ray and CT scan
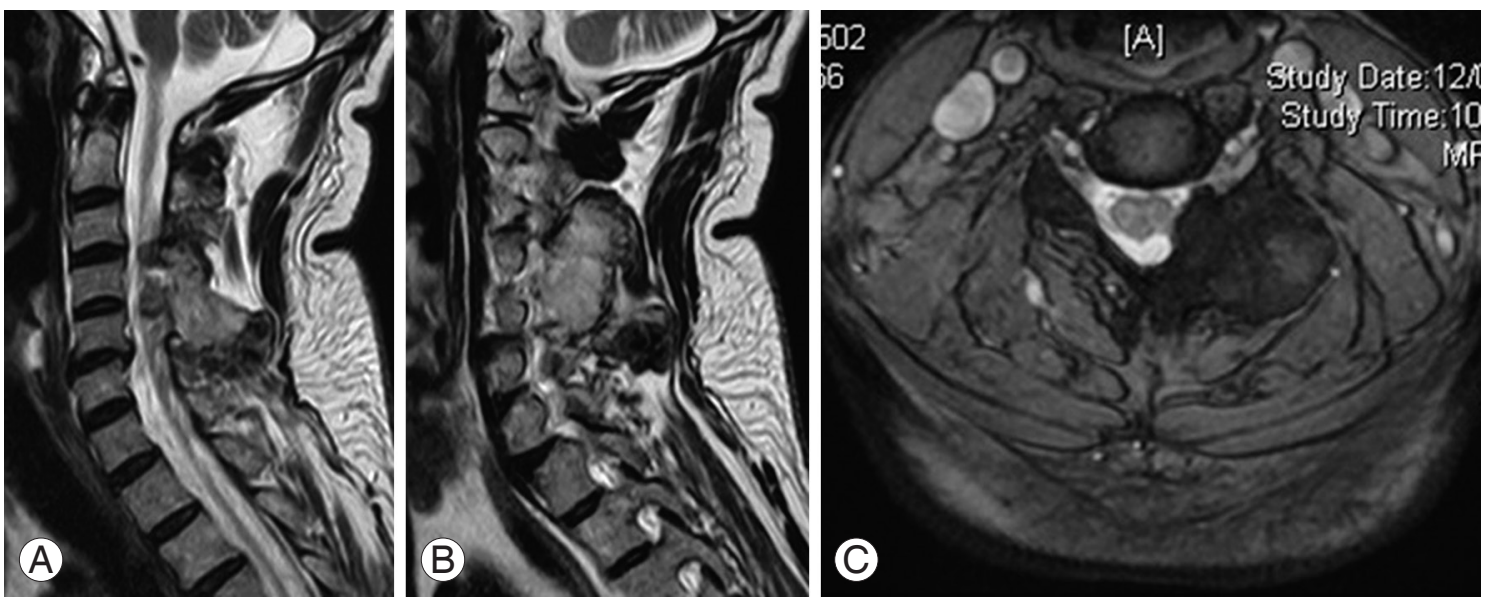

Fig. 3. Magnetic resonance imaging T2 weighted saggital (A), parasaggital (B), and axial (C) images showing the tumor with catilagenous mass and intraspinal extension causing spinal cord compression at C4 and C5.
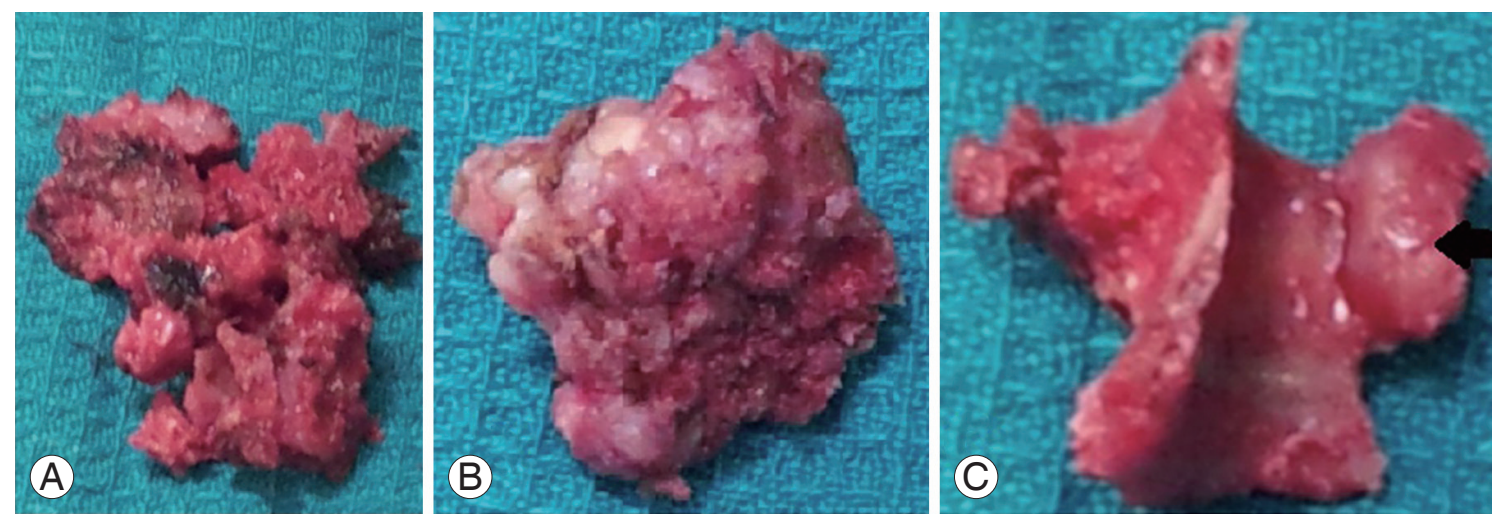

Fig. 4. Gross appearance of the tumor. Osseous part removed piecemeal (A), cartilagenous cap excised enblock (B), lamina of C4 and C5 containing tumor extending into the spinal canal (C).
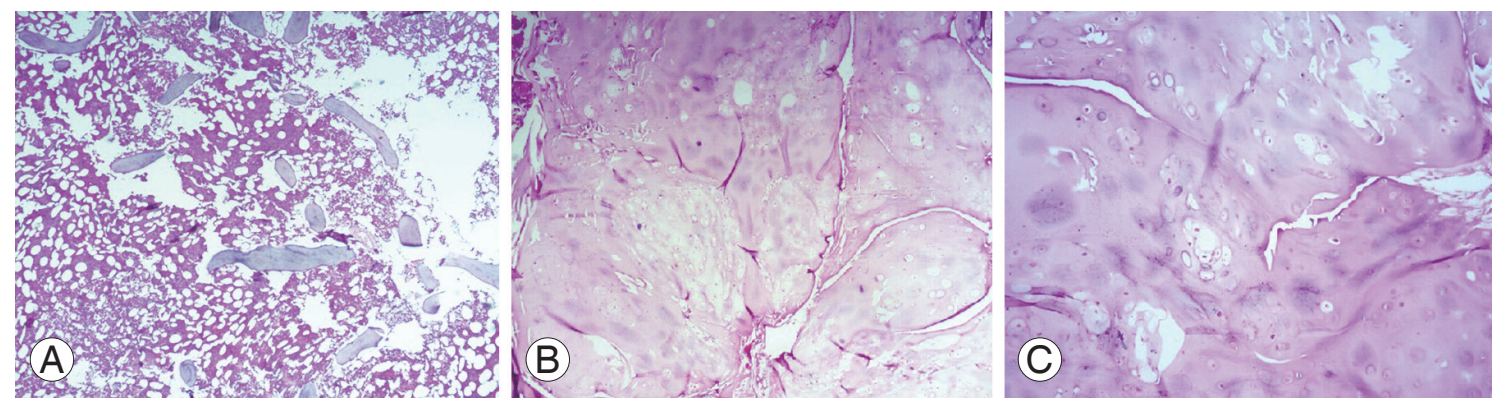

Fig. 5. Histopathological examination of the specimen showing bony lamellae with islands of cartilage (A), cartilagenous tissue under low magnification (B) $(H \& E, \times 10)$, cartilagenous cap under high magnification $(C)(H \& E, \times 40)$. 
showed no recurrence of the tumor (Fig. 7).

\section{Discussion}

Osteochondromas (exostoses) are the most common benign tumors of bone, typically arising from metaphysis of long bones [3]. Exostosis can be either solitary or multiple, with the latter being an autosomal dominant inherited condition [3]. Spinal osteochondromas more commonly are seen in multiple hereditary exostosis (HME) than in solitary form [1]. More than 50\% of spinal osteo- chondromas are located in the cervical spine [1], with the atlantoaxial region being the most common location within the cervical spine [2]. These lesions typically present in the second or third decade of life $[3,5]$ and tend to decrease in size with ageing, due to thinning and gradual disappearance of the cartilage component [1].

Lotfinia et al. [1], in their extensive literature review including 176 spinal osteochondromas, identified 92 lesions located within the cervical spine, of which 48 were the HME sub-type and the remaining 44 were of the solitary form. While none of HME presented after the 5th de-
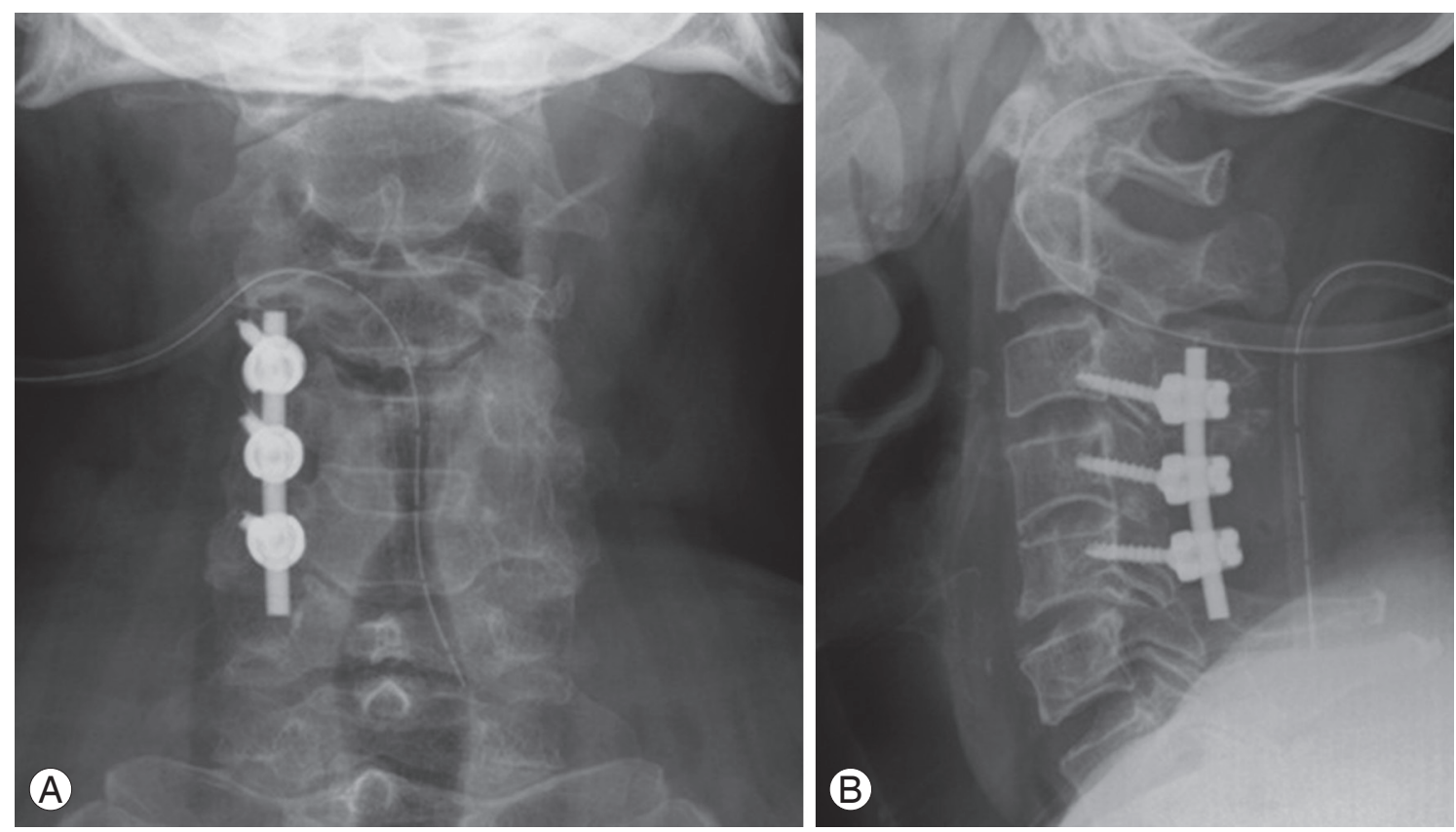

Fig. 6. Postoperative X-ray. Cervical spine anterior-posterior (A) and lateral (B) view showing the tumor excised preserving the facets with lateral mass screw stabilisation on left side.
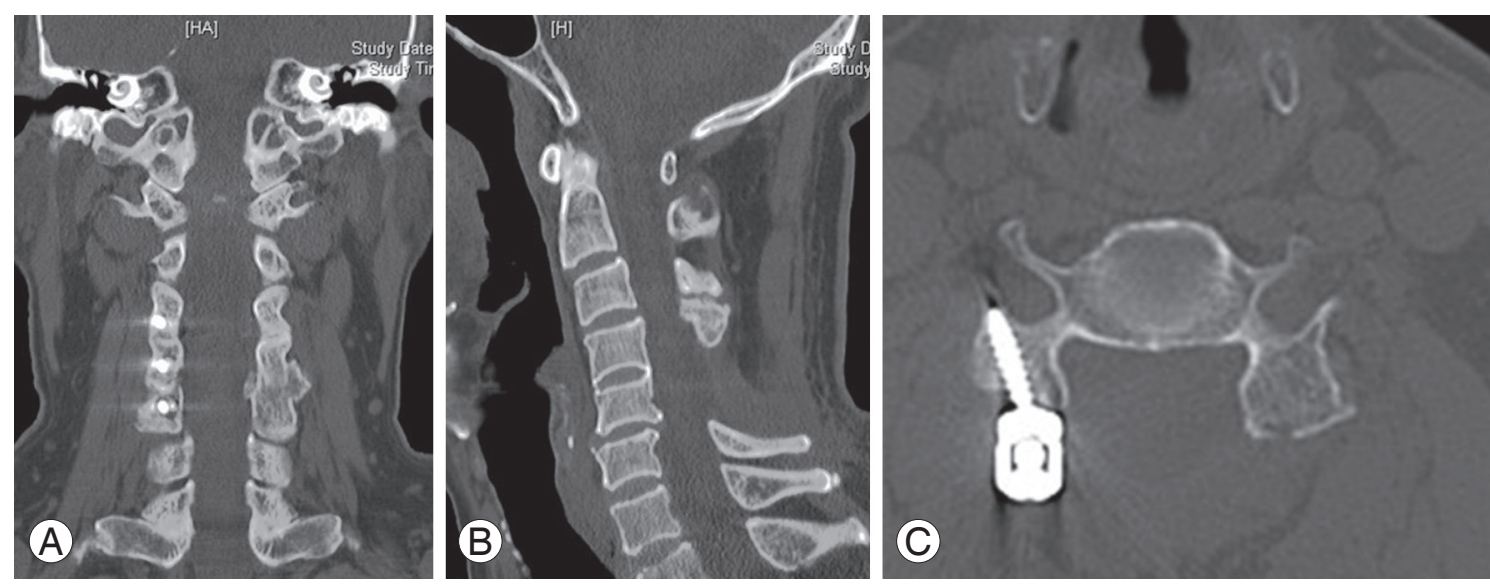

Fig. 7. Computed tomographic scan at follow-up. Coronal (A), saggital (B), and axial (C) images showing spinal canal cleared of the tumor with no evidence of recurrence. 
cade, there were only 6 cases of solitary osteochondromas that originated from the subaxial cervical spine ( $\mathrm{C} 3$ and below) and produced clinical symptoms after the 5th decade of life [1]. Our case is one such rare case of solitary osteochondroma arising from the C4-5 lamina and causing clinical symptoms in late adult life.

The exact reason for the late presentation of spinal exostoses remains unknown. Malignant transformation is a potential, albeit rare cause of increased growth in elderly patients, with a reported incidence of less than $1 \%[3,5]$. Yagi et al. [5] reported 3 cases of spinal osteochondromas in elderly patients and implicated chronic inflammatory conditions such as psoriatic arthritis as the cause for late enlargement of the tumor. Sakai et al. [6] suggested that the onset of associated degenerative changes in the spine was the cause for the late presentation of spinal osteochondromas. This appears to be a plausible explanation in our patient, given that there was no underlying chronic inflammatory condition and that histopathology revealed no malignant transformation.

Osteochondromas arising from the subaxial cervical spine and producing clinical symptoms in late adult life are extremely rare. CT scanning helps in delineating the bony extent of the lesion, while MRI is more sensitive to assessing spinal canal compromise and/or cord compression. A complete surgical excision of the tumor, including the cartilaginous cap as well as the underlying intraspinal component can reliably produce a good clinical outcome as well as minimize the chance of a recurrence. When surgical clearance necessitates sacrificing a significant part of facets compromising the stability, consideration should be given to posterior instrumentation to augment the stability of the cervical spine.

\section{Conflict of Interest}

No potential conflict of interest relevant to this article was reported.

\section{References}

1. Lotfinia I, Vahedi P, Tubbs RS, Ghavame M, Meshkini A. Neurological manifestations, imaging characteristics, and surgical outcome of intraspinal osteochondroma. J Neurosurg Spine 2010;12:474-89.

2. Rao H, Jakheria S. Giant cervical exostosis: a case report with review of literature. J Pediatr Orthop B 2009;18:103-5.

3. Eap C, Litre CF, Noudel R, Duntze J, Theret E, Rousseaux P. Spinal cord compression due to $\mathrm{C} 4$ vertebral arch osteochondroma. Orthop Traumatol Surg Res 2011;97:94-7.

4. Miyakoshi N, Hongo M, Kasukawa Y, Shimada Y. Cervical myelopathy caused by atlas osteochondroma and pseudoarthrosis between the osteochondroma and lamina of the axis: case report. Neurol Med Chir (Tokyo) 2010;50:346-9.

5. Yagi M, Ninomiya K, Kihara M, Horiuchi Y. Symptomatic osteochondroma of the spine in elderly patients. Report of 3 cases. J Neurosurg Spine 2009;11:64-70.

6. Sakai D, Mochida J, Toh E, Nomura T. Spinal osteochondromas in middle-aged to elderly patients. Spine (Phila Pa 1976) 2002;27:E503-6. 